\title{
Prospective evaluation of autoimmune and non-autoimmune subclinical hypothyroidism in Down syndrome children
}

\author{
Giorgia Pepe', Domenico Corica', Luisa De Sanctis², Mariacarolina Salerno ${ }^{3}$, Maria Felicia Faienza, \\ Daniele Tessaris², Gerdi Tuli², Iris Scala5, Laura Penta6 , Angela Alibrandi7, Giovanni Battista Pajno', \\ Tommaso Aversa ${ }^{1}$ and Malgorzata Wasniewska ${ }^{1}$ on behalf of the Thyroid Study Group of the Italian Society of \\ Pediatric Endocrinology and Diabetology (ISPED)
}

${ }^{1}$ Unit of Pediatrics, Department of Human Pathology of Adulthood and Childhood, University of Messina, Messina, Italy, ${ }^{2}$ Division of Pediatric Endocrinology, Department of Public Health and Pediatrics, University of Turin, Regina Margherita Children's Hospital, Turin, Italy, ${ }^{3}$ Department of Translational Medical Sciences, University of Naples Federico II, Naples, Italy, ${ }^{4}$ Department of Biomedical Sciences and Human Oncology, University of Bari, Bari, Italy, ${ }^{5}$ Department of Mother and Child, University of Naples Federico II, Naples, Italy, ${ }^{6}$ Pediatric Clinic, Department of Surgical and Biomedical Sciences, University of Perugia, Perugia, Italy, and 'Department of Economics, University of Messina, Messina, Italy

Correspondence should be addressed to G Pepe Email giorgiapepe23@gmail.com

\begin{abstract}
Objective: To evaluate the prevalence and natural course of autoimmune and non-autoimmune subclinical hypothyroidism (SH) in Down syndrome (DS) children and adolescents.

Design: Prospective multicenter study.

Methods: For the study, 101 DS patients with SH (TSH 5-10 mIU/L; FT4 12-22 pmol/L), aged 2-17 years at SH diagnosis were enrolled. Annual monitoring of TSH, FT4, BMI, height, and L-thyroxine dose was recorded for 5 years. Thyroid autoimmunity was tested at diagnosis and at the end of follow-up.

Results: Thirty-seven out of 101 patients displayed autoantibody positivity (group A); the remaining 64 were classified as non-autoimmune SH (group B). Group A was characterized by higher median age at SH diagnosis and by more frequent family history of thyroid disease ( 6.6 vs 4.7 years, $P=0.001 ; 32.4 \%$ vs $7.8 \%, P=0.001$ respectively), whereas congenital heart defects were more common in group B (65.6\% vs 43.2\%, $P=0.028)$. Gender, median BMI (SDS), height (SDS), FT4, and TSH were similar in both groups. At the end of follow-up: $35.1 \%$ of group A patients developed overt hypothyroidism $(\mathrm{OH})$ vs $17.2 \%$ of group $B(P=0.041) ; 31.25 \%$ of group $B$ vs $10.8 \%$ of group $A$ became biochemically euthyroid $(P=0.02)$; and $37.8 \%$ of group A vs $51.5 \%$ of group B still had SH condition $(P=0.183)$. Logistic regression suggested autoimmunity $(\mathrm{OR}=3.2)$ and baseline $\mathrm{TSH}$ values $(\mathrm{OR}=1.13)$ as predictive factors of the evolution from $\mathrm{SH}$ to $\mathrm{OH}$.

Conclusions: In DS children, non-autoimmune SH showed higher prevalence and earlier onset. The risk of thyroid function deterioration over time seems to be influenced by thyroid autoimmunity and higher baseline TSH values.
\end{abstract}

\section{Introduction}

Subclinical hypothyroidism (SH) is a biochemical condition defined by mildly elevated serum TSH concentrations associated with normal circulating levels of thyroid hormones. (c) 2020 European Society of Endocrinology Printed in Great Britain
Data on $\mathrm{SH}$ in the general pediatric population are scarce; it seems to be a relatively rare condition in children and adolescents (1.7-2.9\%), usually characterized by a self-limiting and remitting process with spontaneous

Published by Bioscientifica Ltd. 
normalization or stabilization of TSH values in the majority of cases (up to $88 \%)(1,2,3)$.

It has already been documented that underlying Hashimoto thyroiditis (HT) can negatively affect the natural course of $\mathrm{SH}$ by increasing the risk of thyroid function deterioration over time. Moreover, the association with chromosomopathies such as Turner syndrome or Down syndrome (DS) may impair the outcome of HT-related SH by further increasing the risk of progression to overt hypothyroidism $(4,5,6)$.

With respect to DS, it is well-known thyroid dysfunction which is much more prevalent and occurs earlier than in the general population. SH is the most common thyroid abnormality in DS children, with prevalence ranging from $25.3 \%$ to $60 \%$ depending on studies $(7,8,9,10)$. The etiology of SH in DS remains still not completely clarified. The most obvious and important hypothesis to explain early onset $\mathrm{SH}$ is based on a generally mild and non-autoimmune thyroid dysfunction, probably caused by a congenital alteration in the regulation of the thyroid gland itself. This finding at group level suggests that it could be in direct relation to the trisomy of chromosome 21 condition $(11,12,13$, $14,15)$. Autoimmunity is also among the hypothesized causes, appearing predominantly from school-age years onward $(9,10,16)$. Indeed, it must be considered that DS children are exposed to an increased risk of autoimmune disorders affecting both endocrine and non-endocrine organs. As a result, HT is one of the most frequent causes of persistent $\mathrm{SH}$, characterized by peculiar features in DS: it is much more common, occurs earlier, without gender predominance, with a more severe biochemical onset and clinical course, and with a higher rate of conversion to Graves disease over time than the general population $(5,17,18)$.

SH in DS children remains relatively unknown because of the scarcity of specific literature data on pathogenesis, natural history, and clinical significance. Consequently, the effectiveness of L-T4 administration and the management of these patients is still controversial $(7,19)$. The American Academy of Pediatrics (AAP) recommends that thyroid function should be evaluated at 6 and 12 months and then annually in all DS children, with increased frequency in SH (20). Despite these guidelines, data showed that up to $25 \%$ of children $>1$ year do not receive recommended screening (21).

Until now, SH in DS appears to be mainly a transient condition, with a rate of evolution toward overt hypothyroidism reported in less than $50 \%$ of cases $(14,16,22)$.
Our prospective study aims to assess relative prevalence and the natural course of autoimmune and non-autoimmune $\mathrm{SH}$ in a large cohort of DS children and adolescents, looking for any predictive factors of spontaneous evolution of thyroid function over time.

\section{Subjects and methods}

\section{Study population}

The study consisted of 101 children and adolescents with DS and $\mathrm{SH}$, who were followed in our outpatient clinics of pediatric endocrinology centers during the period 2013-2018. The following inclusion criteria was carried out: (1.) chronological age $\geq 2$ and $<17$ years at the time of SH diagnosis and (2.) TSH serum levels at SH diagnosis ranging between 5 and $10 \mathrm{mU} / \mathrm{L}$ associated with normal FT4 (reference range 12.0-22.0 pmol/L). DS children with congenital hypothyroidism or early onset isolated hyperthyrotropinemia (in the first 2 years of life) were excluded from the study.

The entire series was divided into two groups, according to whether the etiology of $\mathrm{SH}$ was autoimmune (group A) or non-autoimmune (group B). Therefore, patients of group A were positive for thyroglobulin and/ or thyroid peroxidase serum autoantibodies (TGAbs and TPOAbs respectively), while the autoimmunity profile was entirely negative in group $\mathrm{B}$.

Group A consisted of 37 patients (19 girls and 18 boys), 30 of which were prepubertal and 7 pubertal. Group B consisted of 64 patients ( 30 girls and 34 boys), only one of which was pubertal.

\section{Study design}

SH SD patients who fulfilled the inclusion criteria reported above were enrolled for this prospective multicenter study.

From the time of recruitment, the participants were evaluated every 12 months, for a period of 5 years.

Initial evaluation at $\mathrm{SH}$ diagnosis included: family history for thyroid disease, auxological evaluation (height $\mathrm{H}, \mathrm{BMI}$ ) and pubertal status, presence of congenital heart disease, thyroid function, and autoimmunity tests (TSH, FT4, TGAbs and TPOAbs serum levels). At each annual examination H, BMI, TSH, and FT4 were recorded. Thyroid autoimmunity profile was re-tested at the end of follow-up.

The possible occurrence of other autoimmune diseases was monitored throughout the 5-year observation period. 
In the patients who exhibited an increase in TSH levels above $10 \mathrm{mU} / \mathrm{L}$ and/or a pathological decrease of FT4 levels to $<12 \mathrm{pmol} / \mathrm{L}, \mathrm{L}-\mathrm{T} 4$ treatment was started according to our guidelines (23) and other recommendations $(24,25)$.

In the patients who exhibited a dramatic decrease of TSH serum levels to $<0.3 \mathrm{mIU} / \mathrm{L}$ and/or an increase of FT4 to $>22 \mathrm{pmol} / \mathrm{L}$, methimazole treatment was begun.

According to thyroid function tests, patients were classified into the following biochemical patterns: euthyroidism (both TSH and FT4 within normal limits); SH (normal FT4, as opposed to elevated TSH); overt hypothyroidism, OH (elevated TSH with low FT4); and hyperthyroidism (suppressed TSH, as opposed to either normal or elevated FT4).

\section{Methods}

Serum levels of TSH (reference range 0.3-4.5 mIU/L) and FT4 (reference range 12.0-22.0 pmol/L) were measured by RIA methods. Thyroid autoimmunity was investigated by the measurement of TPOAbs (reference range 0-20 IU/mL) and TGAbs (reference range 0-30 IU/ $\mathrm{mL}$ ) by chemiluminescent immunometric assays. TRABs serum levels (reference range $0-4 \mathrm{IU} / \mathrm{mL}$ ) were measured only in the patients who, during follow-up, displayed a hyperthyroid biochemical evolution and underwent methimazole therapy $(26,27)$.

Auxological assessment was based on $\mathrm{H}$ measurement and BMI calculation. Standing $\mathrm{H}$ was measured with a Harpenden stadiometer (Holtain Ltd, Crymych, Dyfed, UK). BMI was calculated as weight divided by height squared $\left(\mathrm{kg} / \mathrm{m}^{2}\right)$. To allow the comparison between different ages and genders, $\mathrm{H}$ and BMI were expressed as S.D. scores (SDS). Italian specific growth charts for Down syndrome are not available, so the auxological parameters were measured according to the Italian standards assessed by Cacciari et al. (28).

\section{Statistical analysis}

Numerical data were expressed as mean, s.D., median and range; categorical variables were expressed as absolute frequency and percentage.

Non-parametric approach was used since the numerical variables were not normally distributed, such as verified by Kolmogorov Smirnov test.

In order to evaluate the existence of statistically significant differences in the time (basal vs T1, T2, T3, T4, and T5) for each numerical variable, the Wilcoxon test was applied, both for whole sample and for each group (autoimmune and non-autoimmune); for categorical variable 'therapy' the Mc Nemar test was applied. The same analysis was performed within the group of untreated patients.

Statistical comparisons between autoimmune and nonautoimmune patients were performed using Mann Whitney test (for numerical variables) and Chi-Square test or exact Fisher test, when appropriate (for categorical variables).

Univariate logistic regression models were estimated in order to individuate predictive factors of overt hypothyroidism; a multivariate logistic regression model was estimated in order to identify independent predictive factors of overt hypothyroidism.

In order to describe the onset of overt hypothyroidism over time, the Kaplan-Meier curve was made for both groups; moreover, the Log-Rank test was applied to compare the curves (autoimmune vs non-autoimmune).

Statistical analyses were performed using IBM SPSS Statistics for Windows, Version 21. A P-value smaller than 0.05 was considered statistically significant.

The study design was approved by the ethical committee of the University hospital AOU Policlinico 'Gaetano Martino' and by the local ethical committees of the hospitals participating to the study. Informed consent was obtained from the children's parents. The appropriate consent was also obtained earlier from the study group for thyroid diseases of the Italian Society for Pediatric Endocrinology and Diabetology.

\section{Results}

\section{Main data at diagnosis of $\mathrm{SH}$}

The average age at the time of diagnosis of $\mathrm{SH}$ was significantly higher in group A than in group B. Consequently, the prevalence rate of patients who had entered puberty was lower in group B than in group A (Table 1).

A family history of thyroid disease was more common in group A. Instead, a personal history of congenital heart disease was detected more frequently among patients in group B. None of the cardiopathic patients underwent therapy with amiodarone or other drugs which could have affected thyroid function. BMI, height, FT4, and TSH values at follow-up initiation did not significantly differ in these two groups.

Group A was the only group to show any association with other autoimmune diseases: four patients had celiac disease, two patients had diabetes mellitus type 1 , and one patient had vitiligo. 
Table 1 Mean \pm SDS and number $\left(\mathrm{N}^{\circ}\right)$ with percentage (\%) data collected at the start of follow-up in the overall study population of Down syndrome children with subclinical hypothyroidism (SH), in Group A (autoimmune SH) and in Group B (non-autoimmune SH).

\begin{tabular}{l}
\hline \\
\hline$n(\%)$ \\
Gender \\
$\quad$ Female \\
$\quad$ Male \\
Congenital cardiopathy \\
Family history of thyroid disease \\
Age \\
TSH UI/mL \\
FT4 pmol/L \\
H (SDS) \\
BMI (SDS) \\
Pubertal patients \\
Celiac disease \\
DMT1 \\
Vitiligo
\end{tabular}

\begin{tabular}{c}
\hline Total study population \\
\hline $101(100 \%)$ \\
$49(48.5 \%)$ \\
$52(51.5 \%)$ \\
$58(57.4 \%)$ \\
$17(16.8 \%)$ \\
$5.4 \pm 2.9$ \\
$7.4 \pm 4.2$ \\
$15.1 \pm 5.9$ \\
$-1.7 \pm 1.2$ \\
$0.9 \pm 0.9$ \\
$8(7.9 \%)$ \\
$4(4 \%)$ \\
$2(2 \%)$ \\
$1(1 \%)$ \\
\hline
\end{tabular}

\begin{tabular}{c}
\hline Group A \\
\hline $37(36.6 \%)$ \\
$19(51.4 \%)$ \\
$18(48.6 \%)$ \\
$16(43.2 \%)$ \\
$12(32.4 \%)$ \\
$6.6 \pm 3.27$ \\
$8.5 \pm 5.5$ \\
$14.9 \pm 8.0$ \\
$-1.5 \pm 1.5$ \\
$1.1 \pm 0.9$ \\
$7(18.9 \%)$ \\
$4(10.8 \%)$ \\
$2(5.4 \%)$ \\
$1(2.7 \%)$ \\
\hline
\end{tabular}

\begin{tabular}{cccc}
\hline Group B & & $\boldsymbol{P}$ \\
\cline { 1 - 1 } $64(63.4 \%)$ & & \\
$30(46.9 \%)$ & & 0.665 \\
$34(53.1 \%)$ & & 0.665 \\
$42(65.6 \%)$ & & $\mathbf{0 . 0 2 8}$ \\
$5(7.8 \%)$ & & $\mathbf{0 . 0 0 1}$ \\
$4.7 \pm 2.86$ & & $\mathbf{0 . 0 0 1}$ \\
$6.8 \pm 3.1$ & & 0.126 \\
$15.2 \pm 4.0$ & & 0.091 \\
$-1.7 \pm 1.0$ & & 0.247 \\
$0.7 \pm 0.9$ & & 0.12 \\
$1(1.6 \%)$ & & $\mathbf{0 . 0 0 2}$ \\
0 & & $\mathbf{0 . 0 0 4}$ \\
0 & & $\mathbf{0 . 0 4 3}$ \\
0 & & 0.155 \\
\hline
\end{tabular}

$P$ between groups $\mathrm{A}$ and $\mathrm{B}$.

DMT1, diabetes mellitus type $1 ; \mathrm{H}$, height.

\section{Thyroid function patterns at the end of 5-year follow-up}

Overall, 24 out of 101 patients normalized their TSH concentrations $(<5 \mathrm{mUI} / \mathrm{L})$ during the 5 -year observation period, with significantly higher frequency registered among group B compared to group A (31.25\% vs $10.8 \%$, $P=0.02$ ). A deterioration of thyroid function to $\mathrm{OH}$ was observed in 24 patients; this event was instead significantly more frequent in group A than in group B (35.1\% vs $17.2 \%, P=0.004)$ (Fig. 1 ).

The majority of patients in both groups (47 out of 101) maintained, over time, a condition of $\mathrm{SH}(37.8 \%$ in group A vs $51.5 \%$ in group B, $P=0.183$ ).

Only $6(16.2 \%)$ patients in group A and no-one of group B became overtly hyperthyroid, displaying a picture of GD with TRABs positivity and requiring methimazole therapy.

\section{Factors influencing the deterioration of thyroid function over time}

The patients in group A displayed a greater risk of developing overt hypothyroidism in comparison to group $\mathrm{B}(\mathrm{OR}=3.2)$, confirmed by both univariate and multivariate regression model. This suggests that the autoimmune etiology of $\mathrm{SH}$ could be regarded as an independent predictive factor responsible for the deterioration of thyroid function over time. This finding was also highlighted by using the Kaplan-Meier curve (Fig. 2): the progression from $\mathrm{SH}$ to $\mathrm{OH}$ was significantly more frequent among the patients in group A as opposed to the patients in group B $(P=0.011)$.

Moreover, the baseline TSH value was higher in patients who developed overt hypothyroidism; this predictive factor was highlighted by the univariate regression, even thought it was not confirmed by the multivariate regression.

There was no evidence that the others factors involved - gender, age at diagnosis of SH, presence of congenital heart disease, family history for thyroid diseases, baseline auxological parameters and FT4 values - had any influence on the final outcome.

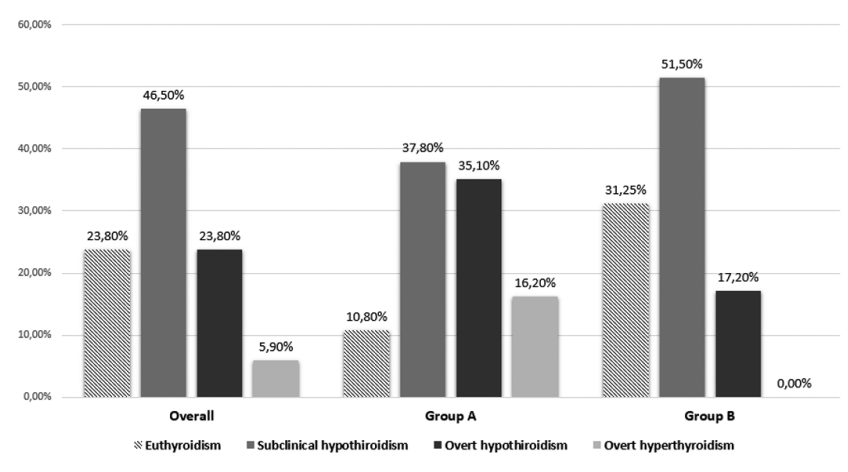

Figure 1

Outcome of subclinical hypothyroidism (SH) at the end of 5-year follow-up: different biochemical patterns of thyroid function detected in Group A (autoimmune $\mathrm{SH}$ ), Group B (non-autoimmune $\mathrm{SH}$ ), and total population (Group $\mathrm{A}+\mathrm{B}$ ). 


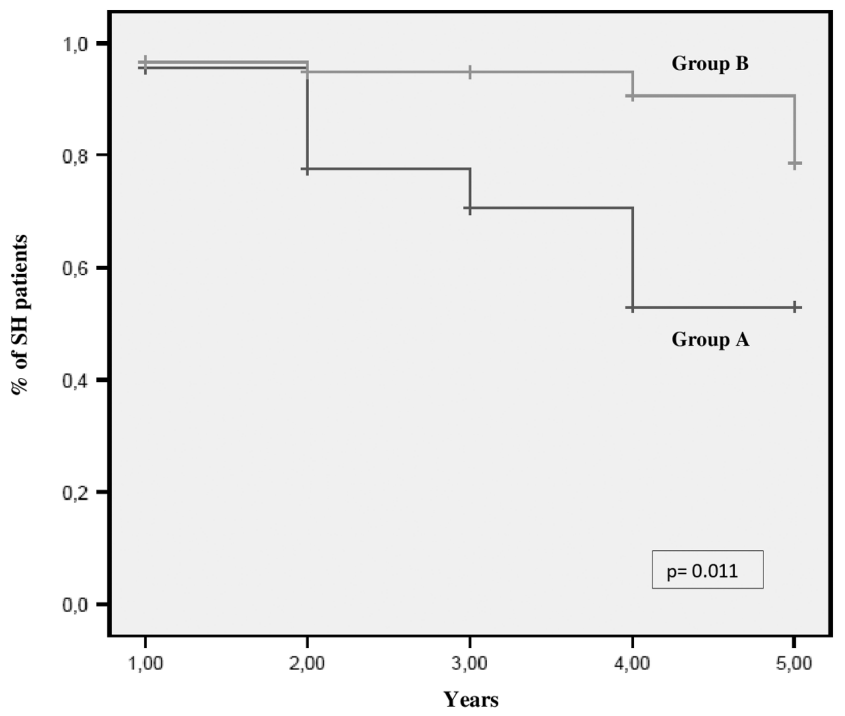

Figure 2

Kaplan-Meier curves showing that the progression from subclinical hypothyroidism (SH) to overt hypothyroidism, during the 5-years follow-up, is significantly more frequent in Group A (autoimmune SH) than in Group B (non-autoimmune $\mathrm{SH}$ ) in Down Syndrome children cohort.

\section{Comparison between treated and untreated patients}

Overall, 48 patients of 101 required L-T4 therapy during the 5-year observation period. The prevalence rate of L-T4 treated patients was higher in group A than in group B (66.6\% vs $33.3 \%)$. The remaining 53 patients had never required replacement therapy during the study protocol; of these, $86.8 \%$ formed part of group B compared to $13.2 \%$ within group A (Table 2).

Among the L-T4 patients treated, the non-autoimmune subgroup portrayed a higher frequency of congenital heart diseases and a lower BMI in at $\mathrm{SH}$ diagnosis, whereas untreated patients displayed a higher H SDS baseline value within the autoimmune subgroup. In addition, we observed a spontaneous and progressive decrease in medium TSH values in untreated patients (Fig. 3).

No significant difference was recorded for the other parameters which are detailed in Table 2.

\section{Discussion}

There are only a few systematic studies on SH in DS and the factors related to its evolution over time are still unclear. To the best of our knowledge, this is the first prospective study aimed to assess annually, over a period of

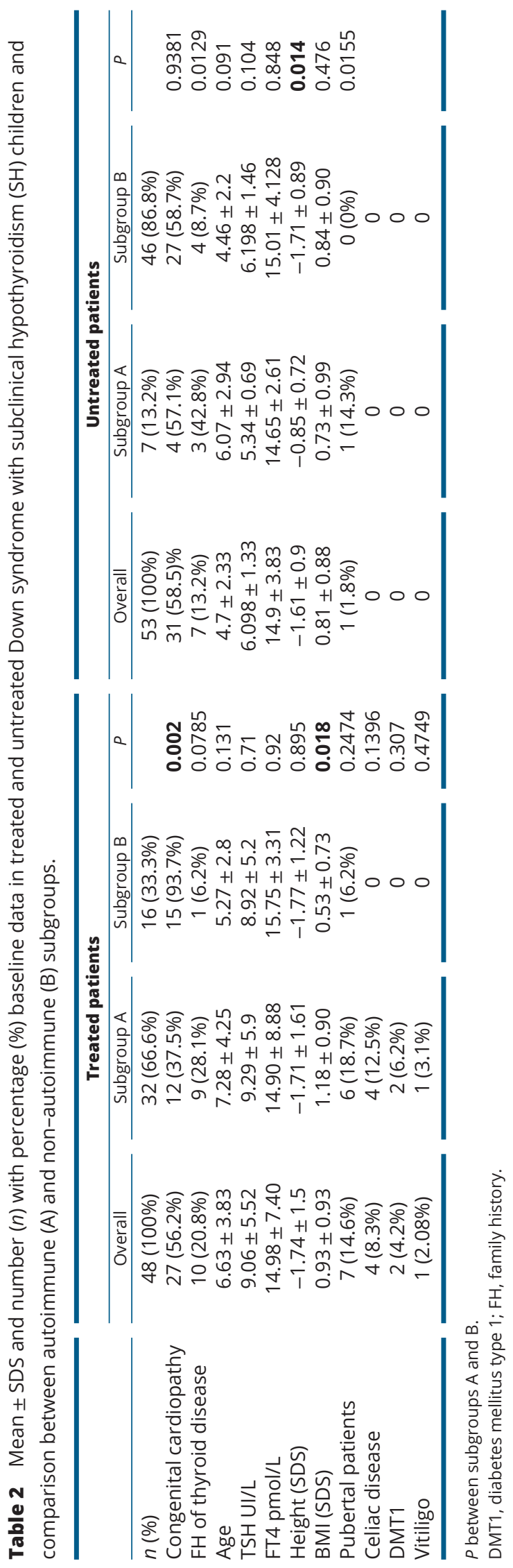

https://eje.bioscientifica.com 


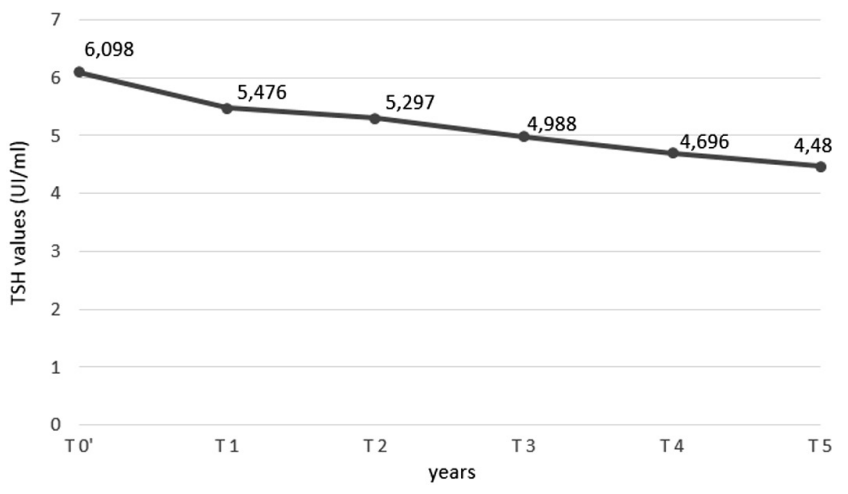

\section{Figure 3}

Medium TSH levels in untreated Down syndrome patients with subclinical hypothyroidism: spontaneous decrease of the values during 5-year follow-up monitoring.

5-years, the natural course of $\mathrm{SH}$ in a large pediatric cohort with DS, excluding congenital hypothyroidism or early onset isolated hyperthyrotropinemia and mainly focusing on the comparison between autoimmune and non-autoimmune etiology of SH.

The first consideration highlighted by our results is that non-autoimmune etiology of $\mathrm{SH}$ is prevalent in DS children (63.4\% of our population never displayed thyroid autoantibodies positivity before $\mathrm{SH}$ diagnosis nor during 5 years of follow-up). This finding is in agreement with previous studies $(8,9,14,22)$. Hence, SH in DS does not appear to be mainly autoimmune-mediated, especially in the first years of life. This phenomenon could be explained by a non-pathological shift in the normal range of TSH as a characteristic of DS, which results in a generally mild and transient form of thyroid disfunction at group level, as suggested by some authors (11, 12, 13, 29). Indeed, the early onset and non-autoimmune SH observed in the majority of DS infants is probably due to a congenital thyroid alteration, which is directly related to the trisomy condition of chromosome 21. Possible mechanisms of isolated elevations in TSH levels can include a delayed maturation of hypothalamic-pituitary-thyroid axis, an inappropriate release of $\mathrm{TSH}$, production of $\mathrm{TSH}$ with lowered activity, TSH insensitivity in the thyroid gland, and hyper-responsiveness to IFN due to over-expression of genes coding for IFN-receptor $(7,14)$.

Another common feature of DS patients is their higher and earlier susceptibility toward autoimmunity than the general population (5). This condition frequently results in HT, which might be regarded as the second most important cause of SH in DS. In particular, autoimmunity plays a greater role much later on, during the school-age years (16). As registered in the present study, the average age of SH diagnosis was higher among patients with autoantibodies. As a consequence of the etiology, patients with autoimmune $\mathrm{SH}$ showed a frequent family history of thyroid disease, and seven of them also developed other autoimmune diseases over time (diabetes mellitus type 1 , celiac disease, and vitiligo).

Congenital heart disease (CHD) was more frequently reported in group $\mathrm{B}$. The association between CHD and thyroid dysfunction is due to the peculiarity of embryonic thyroid development, which is closely associated with the developing heart: the thyroid reaches its position near the base of the neck as a consequence of the heart descent. A disturbance of the proliferation and migration of the neural crest cells, which are also involved in this process, may impair both cardiac and thyroid development (30). These common alterations during embryogenesis of both organs could explain the earlier onset of thyroid dysfunction in non-autoimmune $\mathrm{SH}$ and the higher frequence of congenital heart disease observed in this group.

Therefore, SH in DS may show different patterns of progression. Our study design gave us the opportunity to evaluate whether the association with autoimmune etiology may modify the natural history of SH. According to our results, after a 5-years follow-up period, the most common outcome was the persistence of $\mathrm{SH}$ condition over time, irrespectively of the etiology. SH spontaneous resolution in biochemical euthyroidism was most frequently detected among non-autoimmune patients (31.25\% in group B vs $10.8 \%$ in group A). In contrast, the progression from $\mathrm{SH}$ to $\mathrm{OH}$ occurred more commonly on the basis of autoimmune SH. This finding is clearly confirmed not only by multivariate logistic regression analysis but also by using Kaplan-Meier curve: the onset of $\mathrm{OH}$ is an event registered with significantly higher frequence among the autoimmune group.

In addition, evaluating the features of treated and untreated patients, we mainly recorded that the ones who never required L-T4 therapy displayed a spontaneous and progressive decrease of medium TSH values over time. Finally, only among group A we detected a small percentage $(16.2 \%)$ of patients who became overtly hyperthyroid, displaying a picture of GD with TRABs positivity, which confirmed that DS children might be incline to exhibit a phenotypic metamorphosis from HT to GD and to subsequently fluctuate from hypothyroidism to hyperthyroidism (31).

Overall, our data could confirm that SH in DS is frequently a transitory and benign process, with a rate of evolution toward overt hypothyroidism in less than 
$50 \%$ of cases $(10,13,19)$. Nevertheless, underlying autoimmunity plays an important role and can negatively affect the natural course of $\mathrm{SH}$, with both decreased probabilities of TSH normalization and increased risk of overt thyroid dysfunctions $(5,9,22,32)$.

Moreover, our data highlighted that TSH value at diagnosis was significantly higher in patients who developed a picture of $\mathrm{OH}$; therefore, this predictive factor could be regarded as an early marker of progression into the deterioration of thyroid function.

Based on our results, there was no evidence that the others factors involved - gender, age at diagnosis of $\mathrm{SH}$, presence of congenital heart disease, family history for thyroid diseases, baseline auxological parameters and FT4 values - had any influence on the final outcome.

In conclusion, in DS children: (1.) non-autoimmune SH shows higher prevalence and earlier onset and (2.) the risk of thyroid function deterioration over time, from $\mathrm{SH}$ to $\mathrm{OH}$, seems to be influenced by autoimmune etiology and higher baseline TSH values. Taking into consideration the high frequency of SH in DS and its natural course, a periodic follow-up of thyroid function is recommended in these patients.

\section{Declaration of interest}

The authors declare that there is no conflict of interest that could be perceived as prejudicing the impartiality of this study.

\section{Funding}

This research did not receive any specific grant from any funding agency in the public, commercial, or not-for-profit sector.

\section{References}

1 Wasniewska M, Salerno M, Cassio A, Corrias A, Aversa T, Zirilli G, Capalbo D, Bal M, Mussa A \& De Luca F. Prospective evaluation of the natural course of idiopathic subclinical hypothyroidism in childhood and adolescence. European Journal of Endocrinology 2009 160 417-421. (https://doi.org/10.1530/EJE-08-0625)

2 Wu T, Flowers JW, Tudiver F, Wilson JL \& Punyasavatsut N. Subclinical thyroid disorders and cognitive performance among adolescents in the United States. BMC Pediatrics 20066 12. (https:// doi.org/10.1186/1471-2431-6-12)

3 Lazar L, Frumkin RB, Battat E, Lebenthal Y, Phillip M \& Meyerovitch J. Natural history of thyroid function tests over 5 years in a large pediatric cohort. Journal of Clinical Endocrinology and Metabolism 2009 94 1678-1682. (https://doi.org/10.1210/jc.2008-2615)

4 Wasniewska M, Aversa T, Salerno M, Corrias A, Messina MF, Mussa A, Capalbo D, De Luca F \& Valenzise M. Five-year prospective evaluation of thyroid function in girls with subclinical mild hypothyroidism of different etiology. European Journal of Endocrinology 2015173 801-808. (https://doi.org/10.1530/EJE-15-0484)

5 Aversa T, Valenzise M, Corrias A, Salerno M, De Luca F, Mussa A, Rezzuto M, Lombardo F \& Wasniewska M. Underlying Hashimoto's thyroiditis negatively affects the evolution of subclinical hypothyroidism in children irrespective of other concomitant risk factors. Thyroid 201525 183-187. (https://doi.org/10.1089/ thy.2014.0235)

6 Radetti G, Maselli M, Buzi F, Corrias A, Mussa A, Cambiaso P, Salerno M, Cappa M, Baiocchi M, Gastaldi R et al. The natural history of the normal/mild elevated TSH serum levels in children and adolescents with Hashimoto's thyroiditis and isolated hyperthyrotropinaemia: a 3-year follow-up. Clinical Endocrinology 201276 394-398. (https://doi.org/10.1111/j.13652265.2011.04251.x)

7 Vigone MC, Capalbo D, Weber G \& Salerno M. Mild hypothyroidism in childhood: who, when, and how should be treated? Journal of the Endocrine Society 20182 1024-1039. (https://doi.org/10.1210/js.201700471)

8 Pierce MJ, LaFranchi SH \& Pinter JD. Characterization of thyroid abnormalities in a large cohort of children with Down syndrome

Hormone Research in Paediatrics 201787 170-178. (https://doi. org/10.1159/000457952)

9 Claret C, Goday A, Benaiges D, Chillarón JJ, Flores JA, Hernandez E, Corretger JM \& Cano JF. Subclinical hypothyroidism in the first years of life in patients with Down syndrome. Pediatric Research 201373 674-678. (https://doi.org/10.1038/pr.2013.26)

10 Whooten R, Schmitt J \& Schwartz A. Endocrine Manifestations of Down syndrome. Current Opinion in Endocrinology, Diabetes, and Obesity 201825 61-66. (https://doi.org/10.1097/ MED.0000000000000382)

11 van Trotsenburg AS, Vulsma T, van Rozenburg-Marres SL, van Baar AL, Ridder JC, Heymans HS, Tijssen JG \& de Vijlder JJ. The effect of thyroxine treatment started in the neonatal period on development and growth of two-year-old Down syndrome children: a randomized clinical trial. Journal of Clinical Endocrinology and Metabolism 200590 3304-3311. (https://doi.org/10.1210/jc.20050130)

12 van Trotsenburg AS, Kempers MJ, Endert E, Tijssen JG, de Vijlder JJ \& Vulsma T. Trisomy 21 causes persistent congenital hypothyroidism presumably of thyroidal origin. Thyroid 200616 671-680. (https:// doi.org/10.1089/thy.2006.16.671)

13 Meyerovitch J, Antebi F, Greenberg-Dotan S, Bar-Tal O \& Hochberg Z. Hyperthyrotropinaemia in untreated subjects with Down's syndrome aged 6 months to 64 years: a comparative analysis.Archives of Disease in Childhood 201297 595-598. (https://doi.org/10.1136/ archdischild-2011-300806)

14 Gibson PA, Newton RW, Selby K, Price DA, Leyland K \& Addison GM. Longitudinal study of thyroid function in Down's syndrome in the first two decades. Archives of Disease in Childhood 200590 574-578. (https://doi.org/10.1136/adc.2004.049536)

15 Iughetti L, Lucaccioni L, Fugetto F, Mason A \& Predieri B. Thyroid function in Down syndrome. Expert Review of Endocrinology and Metabolism 201510 525-532. (https://doi.org/10.1586/17446651.201 5.1063995)

16 Karlsson B, Gustafsson J, Hedov G, Ivarsson SA \& Annerén G. Thyroid dysfunction in Down's syndrome: relation to age and thyroid autoimmunity. Archives of Disease in Childhood 199879 242-245. (https://doi.org/10.1136/adc.79.3.242)

17 Aversa T, Salerno M, Radetti G, Faienza MF, Iughetti L, Corrias A, Predieri B, Mussa A, Mirabelli S, De Luca F et al. Peculiarities of presentation and evolution over time of Hashimoto's thyroiditis in children and adolescents with Down's syndrome. Hormones 201514 410-416. (https://doi.org/10.14310/horm.2002.1574)

18 Aversa T, Crisafulli G, Zirilli G, De Luca F, Gallizzi R \& Valenzise M. Epidemiological and clinical aspects of autoimmune thyroid diseases in children with Down's syndrome. Italian Journal of Pediatrics 2018 44 39. (https://doi.org/10.1186/s13052-018-0478-9)

$19 \mathrm{Amr}$ NH. Thyroid disorders in subjects with Down syndrome an update. Acta Bio-Medica 201889 132-139. (https://doi.org/10.23750/ abm.v89i1.7120) 
20 Bull MJ \& Committee on Genetics. Health supervision for children with Down syndrome. Pediatrics 2011128 393-406. (https://doi. org/10.1542/peds.2011-1605)

21 Lavigne J, Sharr C, Elsharkawi I, Ozonoff A, Baumer N, Brasington C, Cannon S, Crissman B, Davidson E, Florez JC et al. Thyroid dysfunction in patients with Down syndrome: results from a multiinstitutional registry study. American Journal of Medical Genetics: Part A 2017173 1539-1545. (https://doi.org/10.1002/ajmg.a.38219)

22 Iughetti L, Predieri B, Bruzzi P, Predieri F, Vellani G, Madeo SF, Garavelli L, Biagioni O, Bedogni G \& Bozzola M. Ten-year longitudinal study of thyroid function in children with Down's syndrome. Hormone Research in Paediatrics 201482 113-121. (https:// doi.org/10.1159/000362450)

23 Arrigo T, Wasniewska M, Crisafulli G, Lombardo F, Messina MF, Rulli I, Salzano G, Valenzise M, Zirilli G \& De Luca F. Subclinical hypothyroidism: the state of the art. Journal of Endocrinological Investigation 200831 79-84. (https://doi.org/10.1007/BF03345571)

24 Cooper DS. Clinical practice. Subclinical hypothyroidism. New England Journal of Medicine 2001345 260-265. (https://doi. org/10.1056/NEJM200107263450406)

25 Lazarus J, Brown RS, Daumerie C, Hubalewska-Dydejczyk A, Negro R \& Vaidya B. 2014 European Thyroid Association guidelines for the management of subclinical hypothyroidism in pregnancy and in children. European Thyroid Journal 20143 76-94. (https://doi. org/10.1159/000362597)

26 Wasniewska M, Corrias A, Salerno M, Mussa A, Capalbo D, Messina MF, Aversa T, Bombaci S, De Luca F \& Valenzise M. Thyroid function patterns at Hashimoto's thyroiditis presentation in childhood and adolescence are mainly conditioned by patients' age. Hormone Research in Paediatrics 201278 232-236. (https://doi. org $/ 10.1159 / 000343815$
27 Wasniewska M, Corrias A, Salerno M, Lombardo F, Aversa T, Mussa A, Capalbo D, De Luca F \& Valenzise M. Outcomes of children with hashitoxicosis. Hormone Research in Paediatrics 201277 36-40. (https://doi.org/10.1159/000334640)

28 Cacciari E, Milani S, Balsamo A, Spada E, Bona G, Cavallo L, Cerutti F, Gargantini L, Greggio N, Tonini G et al. Italian crosssectional growth charts for height, weight and BMI (2 to $20 \mathrm{yr}$ ). Journal of Endocrinological Investigation 200629 581-593. (https://doi. org/10.1007/BF03344156)

29 Kariyawasam D, Carré A, Luton D \& Polak M. Down syndrome and nonautoimmune hypothyroidisms in neonates and infants. Hormone Research in Paediatrics 201583 126-131. (https://doi. org/10.1159/000370004)

30 Olivieri A, Stazi MA, Mastroiacovo P, Fazzini C, Medda E, Spagnolo A, De Angelis S, Grandolfo ME, Taruscio D, Cordeddu V et al. A population-based study on the frequency of additional congenital malformations in infants with congenital hypothyroidism: data from the Italian Registry for Congenital Hypothyroidism (1991-1998). Journal of Clinical Endocrinology and Metabolism 200287 557-562. (https://doi.org/10.1210/jcem.87.2.8235)

31 Aversa T, Valenzise M, Salerno M, Corrias A, Iughetti L, Radetti G, De Luca F \& Wasniewska M. Metamorphic thyroid autoimmunity in Down syndrome: from Hashimoto's thyroiditis to Graves' disease and beyond. Italian Journal of Pediatrics 201541 87. (https://doi. org/10.1186/s13052-015-0197-4)

32 Rubello D, Pozzan GB, Casara D, Girelli ME, Boccato S, Rigon F, Baccichetti C, Piccolo M, Betterle C \& Busnardo B. Natural course of subclinical hypothyroidism in Down's syndrome: prospective study results and therapeutic considerations. Journal of Endocrinological Investigation 199518 35-40. (https://doi. org/10.1007/BF03349694)

Received 15 October 2019

Revised version received 19 January 2020

Accepted 29 January 2020 